\title{
Sufficient conditions of boundedness of L-index and analog of Hayman's Theorem for analytic functions in a ball
}

\author{
Andriy Bandura and Oleh Skaskiv
}

\begin{abstract}
We generalize some criteria of boundedness of $\mathbf{L}$-index in joint variables for analytic in an unit ball functions. Our propositions give an estimate maximum modulus of the analytic function on a skeleton in polydisc with the larger radii by maximum modulus on a skeleton in the polydisc with the lesser radii. An analog of Hayman's Theorem for the functions is obtained. Also we established a connection between class of analytic in ball functions of bounded $l_{j}$-index in every direction $\mathbf{1}_{j}, j \in\{1, \ldots, n\}$ and class of analytic in ball of functions of bounded $\mathbf{L}$-index in joint variables, where $\mathbf{L}(z)=\left(l_{1}(z), \ldots, l_{n}(z)\right), l_{j}: \mathbb{B}^{n} \rightarrow \mathbb{R}_{+}$ is continuous function, $\mathbf{1}_{j}=(0, \ldots, 0, \quad \underbrace{1}, 0, \ldots, 0) \in \mathbb{R}_{+}^{n}, z \in \mathbb{C}^{n}$.

$$
j \text {-th place }
$$
\end{abstract}

Mathematics Subject Classification (2010): 32A05, 32A10, 32A30, 32A40, 30H99.

Keywords: Analytic function, unit ball, bounded L-index in joint variables, maximum modulus, partial derivative, bounded $L$-index in direction.

\section{Introduction}

Recently, there was introduced a concept of analytic function in a ball in $\mathbb{C}^{n}$ of bounded $\mathbf{L}$-index in joint variables [8]. We also obtained criterion of boundedness of L-index in joint variables which describes a local behavior of partial derivatives on a skeleton in the polydisc and established other important properties of analytic functions in a ball of bounded $\mathbf{L}$-index in joint variables. Those investigations used an idea of exhaustion of a ball in $\mathbb{C}^{n}$ by polydiscs.

The presented paper is a continuation of our investigations from [8]. We set the goal to prove new analogues of criteria of boundedness of $\mathbf{L}$-index in joint variables for analytic in a ball functions. Particular, we prove an estimate of maximum modulus on a greater polydisc by maximum modulus on a lesser polydisc (Theorems 3.1, 3.2) and obtain an analog of Hayman's Theorem for analytic functions in a ball of bounded 
L-index in joint variables (Theorems 4.1 and 4.2). For entire functions similar propositions were obtained by A. I. Bandura, M. T. Bordulyak, O. B. Skaskiv $[4,5]$ in a case $\mathbf{L}(z)=\left(l_{1}(z), \ldots, l_{n}(z)\right), z \in \mathbb{C}^{n}$. Also A. I. Bandura, N.V. Petrechko, O. B. Skaskiv $[6,7]$ deduced same results for analytic in a polydisc functions. Hayman's Theorem and its generalizations for different classes of analytic functions $[1,3,5,7,12,15,20,21]$ are very important in theory of functions of bounded index. The criterion is helpful $[1,9]$ to investigate boundedness of index of entire solutions of ordinary or partial differential equations.

Note that the corresponding theorems for entire functions of bounded $l$-index and of bounded $L$-index in direction were also applied to investigate infinite products (see bibliography in $[21,1]$ ). Thus, those generalizations for analytic in a ball functions are necessary to study $\mathbf{L}$-index in joint variables of analytic solutions of PDE's, its systems and multidimensional counterparts of Blaschke products. At the end of the paper, we present a scheme of application of Hayman's Theorem to study properties of analytic solutions in the unit ball.

\section{Main definitions and notations}

We need some standard notations. Denote

$$
\begin{aligned}
& \mathbb{R}_{+}=(0,+\infty), \mathbf{0}=(0, \ldots, 0), \mathbf{1}=(1, \ldots, 1), \\
& \mathbf{1}_{j}=(0, \ldots, 0, \underbrace{1}_{j-\text { th place }}, 0, \ldots, 0) \in \mathbb{R}_{+}^{n}, \\
& R=\left(r_{1}, \ldots, r_{n}\right) \in \mathbb{R}_{+}^{n}, z=\left(z_{1}, \ldots, z_{n}\right) \in \mathbb{C}^{n},|z|=\sqrt{\sum_{j=1}^{n}\left|z_{j}\right|^{2}} .
\end{aligned}
$$

For $A=\left(a_{1}, \ldots, a_{n}\right) \in \mathbb{R}^{n}, B=\left(b_{1}, \ldots, b_{n}\right) \in \mathbb{R}^{n}$ we will use formal notations without violation of the existence of these expressions

$$
\begin{aligned}
& A B=\left(a_{1} b_{1}, \cdots, a_{n} b_{n}\right), A / B=\left(a_{1} / b_{1}, \ldots, a_{n} / b_{n}\right), \\
& A^{B}=a_{1}^{b_{1}} a_{2}^{b_{2}} \cdot \ldots \cdot a_{n}^{b_{n}},\|A\|=a_{1}+\cdots+a_{n},
\end{aligned}
$$

and the notation $A<B$ means that $a_{j}<b_{j}, j \in\{1, \ldots, n\}$; the relation $A \leq B$ is defined similarly. For $K=\left(k_{1}, \ldots, k_{n}\right) \in \mathbb{Z}_{+}^{n}$ denote $K !=k_{1} ! \cdot \ldots \cdot k_{n}$ !. The polydisc $\left\{z \in \mathbb{C}^{n}:\left|z_{j}-z_{j}^{0}\right|<r_{j}, j=1, \ldots, n\right\}$ is denoted by $\mathbb{D}^{n}\left(z^{0}, R\right)$, its skeleton $\left\{z \in \mathbb{C}^{n}:\left|z_{j}-z_{j}^{0}\right|=r_{j}, j=1, \ldots, n\right\}$ is denoted by $\mathbb{T}^{n}\left(z^{0}, R\right)$, and the closed polydisc $\left\{z \in \mathbb{C}^{n}:\left|z_{j}-z_{j}^{0}\right| \leq r_{j}, j=1, \ldots, n\right\}$ is denoted by $\mathbb{D}^{n}\left[z^{0}, R\right]$. The open ball $\left\{z \in \mathbb{C}^{n}:\left|z-z^{0}\right|<r\right\}$ is denoted by $\mathbb{B}^{n}\left(z^{0}, r\right)$, its boundary is a sphere $\mathbb{S}^{n}\left(z^{0}, r\right)=\left\{z \in \mathbb{C}^{n}:\left|z-z^{0}\right|=r\right\}$, the closed ball $\left\{z \in \mathbb{C}^{n}:\left|z-z^{0}\right| \leq r\right\}$ is denoted by $\mathbb{B}^{n}\left[z^{0}, r\right], \mathbb{B}^{n}=\mathbb{B}^{n}(\mathbf{0}, 1), \mathbb{D}=\mathbb{B}^{1}=\{z \in \mathbb{C}:|z|<1\}$.

For $K=\left(k_{1}, \ldots, k_{n}\right) \in \mathbb{Z}_{+}^{n}$ and the partial derivatives of an analytic in $\mathbb{B}^{n}$ function $F(z)=F\left(z_{1}, \ldots, z_{n}\right)$ we use the notation

$$
F^{(K)}(z)=\frac{\partial^{\|K\|} F}{\partial z^{K}}=\frac{\partial^{k_{1}+\cdots+k_{n}} F}{\partial z_{1}^{k_{1}} \ldots \partial z_{n}^{k_{n}}} .
$$

Let $\mathbf{L}(z)=\left(l_{1}(z), \ldots, l_{n}(z)\right)$, where $l_{j}(z): \mathbb{B}^{n} \rightarrow \mathbb{R}_{+}$is a continuous function such that

$$
\left(\forall z \in \mathbb{B}^{n}\right): l_{j}(z)>\beta /(1-|z|), j \in\{1, \ldots, n\}
$$


where $\beta>\sqrt{n}$ is a some constant. For a polydisc A.I. Bandura, N.V. Petrechko and O.B. Skaskiv [6, 7] imposed the restriction $\left(\forall z \in \mathbb{D}^{n}(\mathbf{0}, \mathbf{1})\right): l_{j}(z)>\beta /\left(1-\left|z_{j}\right|\right)$, $j \in\{1, \ldots, n\}$. A similar condition is used in one-dimensional case by S.N. Strochyk, M.M. Sheremeta, V.O. Kushnir $[22,14,21]$.

Note that if $R \in \mathbb{R}_{+}^{n},|R| \leq \beta, z^{0} \in \mathbb{B}^{n}$ and $z \in \mathbb{D}^{n}\left[z^{0}, R / \mathbf{L}\left(z^{0}\right)\right]$ then $z \in \mathbb{B}^{n}$ (see Remark 1 in [8]).

An analytic function $F: \mathbb{B}^{n} \rightarrow \mathbb{C}$ is said to be of bounded $\mathbf{L}$-index (in joint variables), if there exists $n_{0} \in \mathbb{Z}_{+}$such that for all $z \in \mathbb{B}^{n}$ and for all $J \in \mathbb{Z}_{+}^{n}$

$$
\frac{\left|F^{(J)}(z)\right|}{J ! \mathbf{L}^{J}(z)} \leq \max \left\{\frac{\left|F^{(K)}(z)\right|}{K ! \mathbf{L}^{K}(z)}: K \in \mathbb{Z}_{+}^{n},\|K\| \leq n_{0}\right\} .
$$

The least such integer $n_{0}$ is called the $\mathbf{L}$-index in joint variables of the function $F$ and is denoted by $N\left(F, \mathbf{L}, \mathbb{B}^{n}\right)$ (see [8]). Entire and analytic in polydisc functions of bounded $\mathbf{L}$-index in joint variables are considered in $[4,5,6,7,10,13,19,18,16,17]$.

By $Q\left(\mathbb{B}^{n}\right)$ we denote the class of functions $\mathbf{L}$, which satisfy (2.1) and the following condition

$$
\left(\forall R \in \mathbb{R}_{+}^{n},|R| \leq \beta, j \in\{1, \ldots, n\}\right): 0<\lambda_{1, j}(R) \leq \lambda_{2, j}(R)<\infty,
$$

where $\quad \lambda_{1, j}(R)=\inf _{z^{0} \in \mathbb{B}^{n}} \inf \left\{l_{j}(z) / l_{j}\left(z^{0}\right): z \in \mathbb{D}^{n}\left[z^{0}, R / \mathbf{L}\left(z^{0}\right)\right]\right\}$,

$$
\begin{aligned}
& \lambda_{2, j}(R)=\sup _{z^{0} \in \mathbb{B}^{n}} \sup \left\{l_{j}(z) / l_{j}\left(z^{0}\right): z \in \mathbb{D}^{n}\left[z^{0}, R / \mathbf{L}\left(z^{0}\right)\right]\right\} . \\
& \Lambda_{1}(R)=\left(\lambda_{1,1}(R), \ldots, \lambda_{1, n}(R)\right), \Lambda_{2}(R)=\left(\lambda_{2,1}(R), \ldots, \lambda_{2, n}(R)\right) .
\end{aligned}
$$

We need the following results.

Theorem 2.1 ([8]). Let $\mathbf{L} \in Q\left(\mathbb{B}^{n}\right)$. An analytic in $\mathbb{B}^{n}$ function $F$ has bounded $\mathbf{L}$-index in joint variables if and only if for each $R \in \mathbb{R}_{+}^{n},|R| \leq \beta$, there exist $n_{0} \in \mathbb{Z}_{+}, p_{0}>0$ such that for every $z^{0} \in \mathbb{B}^{n}$ there exists $K^{0} \in \mathbb{Z}_{+}^{n},\left\|K^{0}\right\| \leq n_{0}$, and

$$
\max \left\{\frac{\left|F^{(K)}(z)\right|}{K ! \mathbf{L}^{K}(z)}:\|K\| \leq n_{0}, z \in \mathbb{D}^{n}\left[z^{0}, R / \mathbf{L}\left(z^{0}\right)\right]\right\} \leq p_{0} \frac{\left|F^{\left(K^{0}\right)}\left(z^{0}\right)\right|}{K^{0} ! \mathbf{L}^{K^{0}}\left(z^{0}\right)} .
$$

Denote $\widetilde{\mathbf{L}}(z)=\left(\widetilde{l}_{1}(z), \ldots, \widetilde{l}_{n}(z)\right)$. The notation $\mathbf{L} \asymp \widetilde{\mathbf{L}}$ means that there exist

$$
\Theta_{1}=\left(\theta_{1, j}, \ldots, \theta_{1, n}\right) \in \mathbb{R}_{+}^{n}, \Theta_{2}=\left(\theta_{2, j}, \ldots, \theta_{2, n}\right) \in \mathbb{R}_{+}^{n}
$$

such that $\forall z \in \mathbb{B}^{n} \theta_{1, j} \widetilde{l}_{j}(z) \leq l_{j}(z) \leq \theta_{2, j} \widetilde{l}_{j}(z)$ for each $j \in\{1, \ldots, n\}$.

Theorem $2.2([8])$. Let $\mathbf{L} \in Q\left(\mathbb{B}^{n}\right), \mathbf{L} \asymp \widetilde{\mathbf{L}}, \beta\left|\Theta_{1}\right|>\sqrt{n}$. An analytic in $\mathbb{B}^{n}$ function $F$ has bounded $\widetilde{\mathbf{L}}$-index in joint variables if and only if $F$ has bounded $\mathbf{L}$-index in joint variables.

\section{Local behaviour of maximum modulus of analytic in ball function}

For an analytic in $\mathbb{B}^{n}$ function $F$ we put

$$
M\left(r, z^{0}, F\right)=\max \left\{|F(z)|: z \in \mathbb{T}^{n}\left(z^{0}, r\right)\right\}
$$


where $z^{0} \in \mathbb{B}^{n}, r \in \mathbb{R}_{+}^{n}$. Then $M\left(R, z^{0}, F\right)=\max \left\{|F(z)|: z \in \mathbb{D}^{n}\left[z^{0}, R\right]\right\}$, because the maximum modulus for an analytic function in a closed polydisc is attained on its skeleton.

The following proposition uses an idea about the possibility of replacing universal quantifier by existential quantifier in sufficient conditions of index boundedness [2]. To prove an analog of Hayman's Theorem we need this theorem which has an independent interest.

Theorem 3.1. Let $\mathbf{L} \in Q^{n}, F: \mathbb{B}^{n} \rightarrow \mathbb{C}$ be analytic function. If there exist $R^{\prime}$, $R^{\prime \prime} \in \mathbb{R}_{+}^{n}, R^{\prime}<R^{\prime \prime},\left|R^{\prime \prime}\right|<\beta$ and $p_{1}=p_{1}\left(R^{\prime}, R^{\prime \prime}\right) \geq 1$ such that for every $z^{0} \in \mathbb{C}^{n}$

$$
M\left(\frac{R^{\prime \prime}}{\mathbf{L}\left(z^{0}\right)}, z^{0}, F\right) \leq p_{1} M\left(\frac{R^{\prime}}{\mathbf{L}\left(z^{0}\right)}, z^{0}, F\right)
$$

then $F$ is of bounded $\mathbf{L}$-index in joint variables.

Proof. At first, we assume that $\mathbf{0}<R^{\prime}<\mathbf{1}<R^{\prime \prime}$.

Let $z^{0} \in \mathbb{B}^{n}$ be an arbitrary point. We expand a function $F$ in power series

$$
F(z)=\sum_{K \geq \mathbf{0}} b_{K}\left(z-z^{0}\right)^{K}=\sum_{k_{1}, \ldots, k_{n} \geq 0} b_{k_{1}, \ldots, k_{n}}\left(z_{1}-z_{1}^{0}\right)^{k_{1}} \ldots\left(z_{n}-z_{n}^{0}\right)^{k_{n}},
$$

where $b_{K}=b_{k_{1}, \ldots, k_{n}}=\frac{F^{(K)}\left(z^{0}\right)}{K !}$.

Let $\mu\left(R, z^{0}, F\right)=\max \left\{\left|b_{K}\right| R^{K}: K \geq \mathbf{0}\right\}$ be a maximal term of power series (3.2) and

$$
\nu(R)=\nu\left(R, z^{0}, F\right)=\left(\nu_{1}^{0}(R), \ldots, \nu_{n}^{0}(R)\right)
$$

be a set of indices such that

$$
\begin{gathered}
\mu\left(R, z^{0}, F\right)=\left|b_{\nu(R)}\right| R^{\nu(R)}, \\
\|\nu(R)\|=\sum_{j=1}^{n} \nu_{j}(R)=\max \left\{\|K\|: K \geq \mathbf{0},\left|b_{K}\right| R^{K}=\mu\left(R, z^{0}, F\right)\right\} .
\end{gathered}
$$

In view of inequality (3.8) we obtain for any $|R|<1-\left|z^{0}\right|$,

$$
\mu\left(R, z^{0}, F\right) \leq M\left(R, z^{0}, F\right) .
$$

Then for given $R^{\prime}$ and $R^{\prime \prime}$ with $0<\left|R^{\prime}\right|<1<\left|R^{\prime \prime}\right|<\beta$ we conclude

$$
\begin{aligned}
M\left(R^{\prime} R, z^{0}, F\right) & \leq \sum_{k \geq \mathbf{0}}\left|b_{k}\right|\left(R^{\prime} R\right)^{k} \leq \sum_{k \geq \mathbf{0}} \mu\left(R, z^{0}, F\right)\left(R^{\prime}\right)^{k} \\
& =\mu\left(R, z^{0}, F\right) \sum_{k \geq \mathbf{0}}\left(R^{\prime}\right)^{k}=\prod_{j=1}^{n} \frac{1}{1-r_{j}^{\prime}} \mu\left(R, z^{0}, F\right) .
\end{aligned}
$$


Besides,

$$
\begin{aligned}
\ln \mu\left(R, z^{0}, F\right) & =\ln \left\{\left|b_{\nu(R)}\right| R^{\nu(R)}\right\}=\ln \left\{\left|b_{\nu(R)}\right|\left(R R^{\prime \prime}\right)^{\nu(R)} \frac{1}{\left(R^{\prime \prime}\right)^{\nu(R)}}\right\} \\
& =\ln \left\{\left|b_{\nu(R)}\right|\left(R R^{\prime \prime}\right)^{\nu(R)}\right\}+\ln \left\{\frac{1}{\left(R^{\prime \prime}\right)^{\nu(R)}}\right\} \\
& \leq \ln \mu\left(R^{\prime \prime} R, z^{0}, F\right)-\|\nu(R)\| \ln \min _{1 \leq j \leq n} r_{j}^{\prime \prime} .
\end{aligned}
$$

This implies that

$$
\begin{gathered}
\|\nu(R)\| \leq \frac{1}{\ln \min _{1 \leq j \leq n} r_{j}^{\prime \prime}}\left(\ln \mu\left(R^{\prime \prime} R, z^{0}, F\right)-\ln \mu\left(R, z^{0}, F\right)\right) \\
\leq \frac{1}{\ln \min _{1 \leq j \leq n} r_{j}^{\prime \prime}}\left(\ln M\left(R^{\prime \prime} R, z^{0}, F\right)-\ln \left(\prod_{j=1}^{n}\left(1-r_{j}^{\prime}\right) M\left(R^{\prime} R, z^{0}, F\right)\right)\right) \\
\leq \frac{1}{\ln \min _{1 \leq j \leq n} r_{j}^{\prime \prime}}\left(\ln M\left(R^{\prime \prime} R, z^{0}, F\right)-\ln M\left(R^{\prime} R, z^{0}, F\right)\right)-\frac{\sum_{j=1}^{n} \ln \left(1-r_{j}^{\prime}\right)}{\min _{1 \leq j \leq n} r_{j}^{\prime \prime}} \\
=\frac{1}{\min _{1 \leq j \leq n} r_{j}^{\prime \prime}} \ln \frac{M\left(R^{\prime \prime} R, z^{0}, F\right)}{M\left(R^{\prime} R, z^{0}, F\right)}-\frac{\sum_{j=1}^{n} \ln \left(1-r_{j}\right)}{\min _{1 \leq j \leq n} r_{j}^{\prime \prime}} .
\end{gathered}
$$

Put $R=\frac{1}{\mathbf{L}\left(z^{0}\right)}$. Now let $N\left(F, z^{0}, \mathbf{L}\right)$ be the $\mathbf{L}$-index of the function $F$ in joint variables at point $z^{0}$ i. e. it is the least integer for which inequality (2.2) holds at point $z^{0}$. Clearly that

$$
N\left(F, z^{0}, \mathbf{L}\right) \leq \nu\left(\frac{1}{\mathbf{L}\left(z^{0}\right)}, z^{0}, F\right)=\nu\left(R, z^{0}, F\right) .
$$

But

$$
M\left(R^{\prime \prime} / \mathbf{L}\left(z^{0}\right), z^{0}, F\right) \leq p_{1}\left(R^{\prime}, R^{\prime \prime}\right) M\left(R^{\prime} / \mathbf{L}\left(z^{0}\right), z^{0}, F\right) .
$$

Therefore, from (3.3), (3.4), (3.5) we obtain that $\forall z^{0} \in \mathbb{B}^{n}$

$$
N\left(F, z^{0}, \mathbf{L}\right) \leq \frac{-\sum_{j=1}^{2} \ln \left(1-r_{j}^{\prime}\right)}{\ln \min \left\{r_{1}^{\prime \prime}, r_{2}^{\prime \prime}\right\}}+\frac{\ln p_{1}\left(R^{\prime}, R^{\prime \prime}\right)}{\ln \min \left\{r_{1}^{\prime \prime}, r_{2}^{\prime \prime}\right\}}
$$

This means that $F$ has bounded $\mathbf{L}$-index in joint variables, if $\mathbf{0}<R^{\prime}<\mathbf{1}<R^{\prime \prime}$, $\left|R^{\prime \prime}\right|<\beta$.

Now we will prove the theorem for any $\mathbf{0}<R^{\prime}<R^{\prime \prime},\left|R^{\prime \prime}\right|<\beta$. From (3.1) with $\mathbf{0}<R_{1}<R_{2}$ it follows that

$$
\begin{gathered}
\max \left\{|F(z)|: z \in \mathbb{T}^{n}\left(z^{0}, \frac{2 R^{\prime \prime}}{R^{\prime}+R^{\prime \prime}} \frac{R^{\prime}+R^{\prime \prime}}{2 \mathbf{L}\left(z^{0}\right)}\right)\right\} \\
\leq P_{1} \max \left\{|F(z)|: z \in \mathbb{T}^{n}\left(z^{0}, \frac{2 R^{\prime}}{R^{\prime}+R^{\prime \prime}} \frac{R^{\prime}+R^{\prime \prime}}{2 \mathbf{L}\left(z^{0}\right)}\right)\right\} .
\end{gathered}
$$


Denoting $\widetilde{\mathbf{L}}(z)=\frac{2 \mathbf{L}(z)}{R^{\prime}+R^{\prime \prime}}$, we obtain

$$
\begin{gathered}
\max \left\{|F(z)|: z \in \mathbb{T}^{n}\left(z^{0}, \frac{2 R^{\prime \prime}}{\left(R^{\prime}+R^{\prime \prime}\right) \widetilde{\mathbf{L}}\left(z^{0}\right)}\right)\right\} \\
\leq P_{1} \max \left\{|F(z)|: z \in \mathbb{T}^{n}\left(z^{0}, \frac{2 R^{\prime \prime}}{\left(R^{\prime}+R^{\prime \prime}\right) \widetilde{\mathbf{L}}\left(z^{0}\right)}\right)\right\},
\end{gathered}
$$

where $\mathbf{0}<\frac{2 R^{\prime}}{R^{\prime}+R^{\prime \prime}}<\mathbf{1}<\frac{2 R^{\prime \prime}}{R^{\prime}+R^{\prime \prime}}$. Taking into account the first part of the proof, we conclude that the function $F$ has bounded $\widetilde{\mathbf{L}}$-index in joint variables. By Theorem 2.2, the function $F$ is of bounded $\mathbf{L}$-index in joint variables.

Also the corresponding necessary conditions are valid.

Theorem 3.2. Let $\mathbf{L} \in Q\left(\mathbb{B}^{n}\right)$. If an analytic in $\mathbb{B}^{n}$ function $F$ has bounded $\mathbf{L}$-index in joint variables then for any $R^{\prime}, R^{\prime \prime} \in \mathbb{R}_{+}^{n}, R^{\prime}<R^{\prime \prime},\left|R^{\prime \prime}\right| \leq \beta$, there exists a number $p_{1}=p_{1}\left(R^{\prime}, R^{\prime \prime}\right) \geq 1$ such that for every $z^{0} \in \mathbb{B}^{n}$ inequality (3.1) holds.

Proof. Let $N(F, \mathbf{L})=N<+\infty$. Suppose that inequality (3.1) does not hold i.e. there exist $R^{\prime}, R^{\prime \prime}, 0<\left|R^{\prime}\right|<\left|R^{\prime \prime}\right|<\beta$, such that for each $p_{*} \geq 1$ and for some $z^{0}=z^{0}\left(p_{*}\right)$

$$
M\left(\frac{R^{\prime \prime}}{\mathbf{L}\left(z^{0}\right)}, z^{0}, F\right)>p_{*} M\left(\frac{R^{\prime}}{\mathbf{L}\left(z^{0}\right)}, z^{0}, F\right) .
$$

By Theorem 2.1, there exists a number $p_{0}=p_{0}\left(R^{\prime \prime}\right) \geq 1$ such that for every $z^{0} \in \mathbb{B}^{n}$ and some $K^{0} \in \mathbb{Z}_{+}^{n},\left\|K^{0}\right\| \leq N$, (i.e. $n_{0}=N$, see proof of necessity of Theorem 2.1 in [8]) one has

We put

$$
M\left(\frac{R^{\prime \prime}}{\mathbf{L}\left(z^{0}\right)}, z^{0}, F^{\left(K^{0}\right)}\right) \leq p_{0}\left|F^{\left(K^{0}\right)}\left(z^{0}\right)\right| .
$$

$$
\begin{gathered}
b_{1}=p_{0}\left(\prod_{j=2}^{n} \lambda_{2, j}^{N}\left(R^{\prime \prime}\right)\right)(N !)^{n-1}\left(\sum_{j=1}^{N} \frac{(N-j) !}{\left(r_{1}^{\prime \prime}\right)^{j}}\right)\left(\frac{r_{1}^{\prime \prime} r_{2}^{\prime \prime} \ldots r_{n}^{\prime \prime}}{r_{1}^{\prime} r_{2}^{\prime} \ldots r_{n}^{\prime}}\right)^{N} \\
b_{2}=p_{0}\left(\prod_{j=3}^{n} \lambda_{2, j}^{N}\left(R^{\prime \prime}\right)\right)(N !)^{n-2}\left(\sum_{j=1}^{N} \frac{(N-j) !}{\left(r_{2}^{\prime \prime}\right)^{j}}\right)\left(\frac{r_{2}^{\prime \prime} \ldots r_{n}^{\prime \prime}}{r_{2}^{\prime} \ldots r_{n}^{\prime}}\right)^{N}\left\{1, \frac{1}{\left(r_{1}^{\prime}\right)^{N}}\right\} \\
b_{n-1}=p_{0} \lambda_{2, n}^{N}\left(R^{\prime}\right) N !\left(\sum_{j=1}^{N} \frac{(N-j) !}{\left(r_{n-1}^{\prime \prime}\right)^{j}}\right)\left(\frac{r_{n-1}^{\prime \prime} r_{n}^{\prime \prime}}{r_{n-1}^{\prime} r_{n}^{\prime}}\right)^{N} \max \left\{1, \frac{1}{\left(r_{1}^{\prime} \ldots r_{n-2}^{\prime}\right)^{N}}\right\}, \\
b_{n}=p_{0}\left(\sum_{j=1}^{N} \frac{(N-j) !}{\left(r_{n}^{\prime \prime}\right)^{j}}\right)\left(\frac{r_{n}^{\prime \prime}}{r_{n}^{\prime}}\right)^{N} \max \left\{1, \frac{1}{\left(r_{1}^{\prime} \ldots r_{n-1}^{\prime}\right)^{N}}\right\}
\end{gathered}
$$

and

$$
p_{*}=(N !)^{n} p_{0}\left(\frac{r_{1}^{\prime \prime} r_{2}^{\prime \prime} \ldots r_{n}^{\prime \prime}}{r_{1}^{\prime} r_{2}^{\prime} \ldots r_{n}^{\prime}}\right)^{N}+\sum_{k=1}^{n} b_{k}+1 .
$$


Let $z^{0}=z^{0}\left(p_{*}\right)$ be a point for which inequality (3.6) holds and $K^{0}$ be such that (3.7) holds and

$$
M\left(\frac{R^{\prime}}{\mathbf{L}\left(z^{0}\right)}, z^{0}, F\right)=\left|F\left(z^{*}\right)\right|, M\left(\frac{r^{\prime \prime}}{\mathbf{L}\left(z^{0}\right)}, z^{0}, F^{(J)}\right)=\left|F^{(J)}\left(z_{J}^{*}\right)\right|
$$

for every $J \in \mathbb{Z}_{+}^{n},\|J\| \leq N$. We apply Cauchy's inequality

$$
\left|F^{(J)}\left(z^{0}\right)\right| \leq J !\left(\frac{\mathbf{L}\left(z^{0}\right)}{R^{\prime}}\right)^{J}\left|F\left(z^{*}\right)\right|
$$

for estimate the difference

$$
\begin{aligned}
& \left|F^{(J)}\left(z_{J, 1}^{*}, z_{J, 2}^{*}, \ldots, z_{J, n}^{*}\right)-F^{(J)}\left(z_{1}^{0}, z_{J, 2}^{*}, \ldots, z_{J, n}^{*}\right)\right| \\
& \quad=\left|\int_{z_{1}^{0}}^{z_{J, 1}^{*}} \frac{\partial^{\|J\|+1} F}{\partial z_{1}^{j_{1}+1} \partial z_{2}^{j_{2}} \ldots \partial z_{n}^{j_{n}}}\left(\xi, z_{J, 2}^{*}, \ldots, z_{J, n}^{*}\right) d \xi\right| \\
& \quad \leq\left|\frac{\partial^{\|J\|+1} F}{\partial z_{1}^{j_{1}+1} \partial z_{2}^{j_{2}} \ldots \partial z_{n}^{j_{n}}}\left(z_{\left(j_{1}+1, j_{2}, \ldots, j_{n}\right)}^{*}\right)\right| \frac{r_{1}^{\prime \prime}}{l_{1}\left(z^{0}\right)} .
\end{aligned}
$$

Taking into account $\left(z_{1}^{0}, z_{J, 2}^{*}, \ldots, z_{J, n}^{*}\right) \in \mathbb{D}^{n}\left[z^{0}, \frac{R^{\prime \prime}}{\mathbf{L}\left(z^{0}\right)}\right]$, for all $k \in\{1, \ldots, n\}$,

$$
\left|z_{J, k}^{*}-z_{k}^{0}\right|=\frac{r_{k}^{\prime \prime}}{l_{k}\left(z^{0}\right)}, l_{k}\left(z_{1}^{0}, z_{J, 2}^{*}, \ldots, z_{J, n}^{*}\right) \leq \lambda_{2, k}\left(R^{\prime \prime}\right) l_{k}\left(z^{0}\right)
$$

and (3.8) with $J=K^{0}$, by Theorem 2.1 we have

$$
\begin{aligned}
& \left|F^{(J)}\left(z_{1}^{0}, z_{J, 2}^{*}, \ldots, z_{J, n}^{*}\right)\right| \\
& \leq \frac{J ! l_{1}^{j_{1}}\left(z_{1}^{0}, z_{J, 2}^{*}, \ldots, z_{J, n}^{*}\right) \prod_{k=2}^{n} l_{k}^{j_{k}}\left(z_{1}^{0}, z_{J, 2}^{*}, \ldots, z_{J, n}^{*}\right)}{K^{0} ! \mathbf{L}^{K^{0}}\left(z^{0}\right)} p_{0}\left|F^{\left(K^{0}\right)}\left(z^{0}\right)\right| \\
& \leq \frac{J ! \mathbf{L}^{J}\left(z^{0}\right) \prod_{k=2}^{n} \lambda_{2, k}^{j_{k}}\left(R^{\prime \prime}\right)}{K^{0} ! \mathbf{L}^{K^{0}}\left(z^{0}\right)} p_{0} K^{0} !\left(\frac{\mathbf{L}\left(z^{0}\right)}{R^{\prime}}\right)^{K^{0}}\left|F\left(z^{*}\right)\right| \\
& =\frac{p_{0} J ! \mathbf{L}^{J}\left(z^{0}\right) \prod_{k=2}^{n} \lambda_{2, k}^{j_{k}}\left(R^{\prime \prime}\right)}{\left(R^{\prime}\right)^{K^{0}}}\left|F\left(z^{*}\right)\right| \text {. }
\end{aligned}
$$

From inequalities (3.9) and (3.10) it follows that

$$
\begin{gathered}
\left|\frac{\partial^{\|J\|+1} F}{\partial z_{1}^{j_{1}+1} \partial z_{2}^{j_{2}} \ldots \partial z_{n}^{j_{n}}}\left(z_{\left(j_{1}+1, j_{2}, \ldots, j_{n}\right)}^{*}\right)\right| \\
\geq \frac{l_{1}\left(z^{0}\right)}{r_{1}^{\prime \prime}}\left\{\left|F^{(J)}\left(z_{j}^{*}\right)\right|-\left|F^{(J)}\left(z_{1}^{0}, z_{J, 2}^{*}, \ldots, z_{J, n}^{*}\right)\right|\right\} \\
\geq \frac{l_{1}\left(z_{1}^{0}\right)}{r_{1}^{\prime \prime}}\left|F^{(J)}\left(z_{j}^{*}\right)\right|-\frac{p_{0} J ! \mathbf{L}^{\left(j_{1}+1, j_{2}, \ldots, j_{n}\right)}\left(z^{0}\right) \prod_{k=2}^{n} \lambda_{2, k}^{j_{k}}\left(R^{\prime \prime}\right)}{r_{1}^{\prime \prime}\left(R^{\prime}\right)^{K^{0}}}\left|F\left(z^{*}\right)\right| .
\end{gathered}
$$

Then

$$
\left|F^{\left(K^{0}\right)}\left(z_{K^{0}}^{*}\right)\right| \geq \frac{l_{1}\left(z^{0}\right)}{r_{1}^{\prime \prime}}\left|\frac{\partial^{\left\|K^{0}\right\|-1} f}{\partial z_{1}^{k_{1}^{0}-1} \partial z_{2}^{k_{2}^{0}} \ldots \partial z_{n}^{k_{n}^{0}}}\left(z_{\left(k_{1}^{0}-1, k_{2}^{0}, \ldots, k_{n}^{0}\right)}^{*}\right)\right|
$$




$$
\begin{aligned}
& -\frac{p_{0}\left(k_{1}^{0}-1\right) ! k_{2}^{0} ! \ldots k_{n}^{0} ! \mathbf{L}^{K^{0}}\left(z^{0}\right) \prod_{i=2}^{n} \lambda_{2, i}^{k_{i}^{0}}\left(R^{\prime \prime}\right)}{r_{1}^{\prime \prime}\left(R^{\prime}\right)^{K^{0}}}\left|F\left(z^{*}\right)\right| \\
& \geq \frac{l_{1}^{2}\left(z^{0}\right)}{\left(r_{1}^{\prime \prime}\right)^{2}}\left|\frac{\partial^{\left\|K^{0}\right\|-2} f}{\partial z_{1}^{k_{1}^{0}-2} \partial z_{2}^{k_{2}^{0}} \ldots \partial z_{n}^{k_{n}^{0}}}\left(z_{\left(k_{1}^{0}-2, k_{2}^{0}, \ldots, k_{n}^{0}\right)}^{*}\right)\right| \\
& -\frac{p_{0}\left(k_{1}^{0}-2\right) ! k_{2}^{0} ! \ldots k_{n}^{0} ! \mathbf{L}^{K^{0}}\left(z^{0}\right) \prod_{i=2}^{n} \lambda_{2, i}^{k_{i}^{0}}\left(R^{\prime \prime}\right)}{\left(r_{1}^{\prime \prime}\right)^{2}\left(R^{\prime}\right)^{K^{0}}}\left|F\left(z^{*}\right)\right| \\
& -\frac{p_{0}\left(k_{1}^{0}-1\right) ! k_{2}^{0} ! \ldots k_{n}^{0} ! \mathbf{L}^{K^{0}}\left(z^{0}\right) \prod_{i=2}^{n} \lambda_{2, i}^{k_{i}^{0}}\left(r_{i}^{\prime \prime}\right)}{r_{1}^{\prime \prime}\left(R^{\prime}\right)^{K^{0}}}\left|F\left(z^{*}\right)\right| \\
& \geq \frac{l_{1}^{k_{1}^{0}}\left(z^{0}\right)}{\left(r_{1}^{\prime \prime}\right)_{1}^{k_{1}^{0}}}\left|\frac{\partial^{\left\|K^{0}\right\|-k_{1}^{0}} f}{\partial z_{2}^{k_{2}^{0}} \ldots \partial z_{n}^{k_{n}^{0}}}\left(z_{\left(0, k_{2}^{0}, \ldots, k_{n}^{0}\right)}^{*}\right)\right| \\
& -\frac{p_{0}}{\left(R^{\prime}\right)^{K^{0}}} \mathbf{L}^{K^{0}}\left(z^{0}\right)\left(\prod_{i=2}^{n} \lambda_{2, i}^{k_{i}^{0}}\left(R^{\prime \prime}\right)\right) k_{2}^{0} ! \ldots k_{n}^{0} ! \sum_{j_{1}=1}^{k_{1}^{0}} \frac{\left(k_{1}^{0}-j_{1}\right) !}{\left(r_{1}^{\prime \prime}\right)^{j_{1}}}\left|F\left(z^{*}\right)\right| \ldots \\
& \geq \frac{l_{1}^{k_{1}^{0}}\left(z^{0}\right)}{\left(r_{1}^{\prime \prime}\right)_{1}^{k_{1}^{0}}} \frac{l_{2}^{k_{2}^{0}}\left(z^{0}\right)}{\left(r_{2}^{\prime \prime}\right)^{k_{2}^{0}}}\left|\frac{\partial^{\left\|K^{0}\right\|-k_{1}^{0}-k_{2}^{0} f}}{\partial z_{3}^{k_{3}^{0}} \ldots \partial z_{n}^{k_{n}^{0}}}\left(z_{\left(0,0, k_{3}^{0}, \ldots, k_{n}^{0}\right)}^{*}\right)\right| \\
& -\frac{l_{1}^{k_{1}^{0}}\left(z^{0}\right) p_{0} L^{\left(0, k_{2}^{0}, \ldots, k_{n}^{0}\right)}\left(z^{0}\right)}{\left(r_{1}^{\prime \prime}\right)^{k_{1}^{0}}\left(R^{\prime}\right)^{K^{0}}}\left(\prod_{i=3}^{n} \lambda_{2, i}^{k_{i}^{0}}\left(R^{\prime \prime}\right)\right) k_{3}^{0} ! \ldots k_{n}^{0} ! \sum_{i_{2}=1}^{k_{2}^{0}} \frac{\left(k_{2}^{0}-j_{2}\right) !}{\left(r_{2}^{\prime \prime}\right)^{j_{2}}}\left|F\left(z^{*}\right)\right| \\
& -\frac{p_{0}}{\left(R^{\prime}\right)^{K^{0}}} \mathbf{L}^{K^{0}}\left(z^{0}\right)\left(\prod_{i=2}^{n} \lambda_{2, i}^{k_{i}^{0}}\left(R^{\prime \prime}\right)\right) k_{2}^{0} ! \ldots k_{n}^{0} ! \sum_{j_{1}=1}^{k_{1}^{0}} \frac{\left(k_{1}^{0}-j_{1}\right) !}{\left(r_{1}^{\prime \prime}\right)^{j_{1}}}\left|F\left(z^{*}\right)\right| \\
& \geq\left(\frac{L\left(z^{0}\right)}{R^{\prime \prime}}\right)\left|F\left(z_{0}^{*}\right)\right|-\left|F\left(z^{*}\right)\right| \sum_{i=1}^{b} \tilde{b}_{i},
\end{aligned}
$$

where in view of the inequalities $\lambda_{2, i}\left(R^{\prime \prime}\right) \geq 1$ and $R^{\prime \prime} \geq R^{\prime}$ we have

$$
\begin{gathered}
\tilde{b}_{1}=\frac{p_{0}}{\left(R^{\prime}\right)^{K^{0}}} \mathbf{L}^{K^{0}}\left(z^{0}\right)\left(\prod_{i=2}^{n} \lambda_{2, i}^{k_{i}^{0}}\left(R^{\prime \prime}\right)\right) k_{2}^{0} ! \ldots k_{n}^{0} ! \sum_{j_{1}=1}^{k_{1}^{0}} \frac{\left(k_{1}^{0}-j_{1}\right) !}{\left(r_{1}^{\prime \prime}\right)^{j_{1}}} \\
=\left(\frac{\mathbf{L}\left(z^{0}\right)}{R^{\prime \prime}}\right)^{K^{0}}\left(\frac{R^{\prime \prime}}{R^{\prime}}\right)^{K^{0}} p_{0}\left(\prod_{i=2}^{n} \lambda_{2, i}^{k_{i}^{0}}\left(R^{\prime \prime}\right)\right) k_{2}^{0} ! \ldots k_{n}^{0} ! \sum_{j_{1}=1}^{k_{1}^{0}} \frac{\left(k_{1}^{0}-j_{1}\right) !}{\left(r_{1}^{\prime \prime}\right)^{j_{1}}} \leq\left(\frac{\mathbf{L}\left(z^{0}\right)}{R^{\prime \prime}}\right)^{K^{0}} b_{1}, \\
\tilde{b}_{2}=\frac{p_{0}}{\left(R^{\prime}\right)^{K^{0}}} \mathbf{L}^{K^{0}}\left(z^{0}\right)\left(\prod_{i=3}^{n} \lambda_{2, i}^{k_{i}^{0}}\left(R^{\prime \prime}\right)\right) \frac{k_{3}^{0} ! \ldots k_{n}^{0} !}{\left(r_{1}^{\prime \prime}\right)^{k_{1}^{0}}} \sum_{j_{2}=1}^{k_{2}^{0}} \frac{\left(k_{2}^{0}-j_{2}\right) !}{\left(r_{2}^{\prime \prime}\right)^{j_{2}}} \leq\left(\frac{\mathbf{L}\left(z^{0}\right)}{R^{\prime \prime}}\right)^{K^{0}} b_{2},
\end{gathered}
$$




$$
\begin{aligned}
& \tilde{b}_{n-1}=\frac{p_{0}}{\left(R^{\prime}\right)^{K^{0}}} \mathbf{L}^{K^{0}}\left(z^{0}\right) \lambda_{2, n}^{k_{n}^{0}}\left(R^{\prime \prime}\right) \frac{k_{n}^{0} !}{\left(r_{1}^{\prime \prime}\right)^{k_{1}^{0}} \ldots\left(r_{n-2}^{\prime \prime}\right)^{k_{n-2}^{0}}} \times \\
& \times \sum_{j_{n-1}=1}^{k_{n-1}^{0}} \frac{\left(k_{n-1}^{0}-j_{n-1}\right) !}{\left(r_{n-1}^{\prime \prime}\right)^{j_{n-1}}} \leq\left(\frac{\mathbf{L}\left(z^{0}\right)}{R^{\prime \prime}}\right)^{K^{0}} b_{n-1} \\
& \tilde{b}_{n}=\frac{p_{0}}{\left(R^{\prime}\right)^{K^{0}}} \mathbf{L}^{K^{0}}\left(z^{0}\right) \frac{1}{\left(r_{1}^{\prime \prime}\right)^{k_{1}^{0}} \ldots\left(r_{n-1}^{\prime \prime}\right)^{k_{n-1}^{0}}} \sum_{j_{n}=1}^{k_{n}^{0}} \frac{\left(k_{n}^{0}-j_{n}\right) !}{\left(r_{n}^{\prime \prime}\right)^{j_{n}}} \leq\left(\frac{\mathbf{L}\left(z^{0}\right)}{R^{\prime \prime}}\right)^{K^{0}} b_{n} .
\end{aligned}
$$

Thus, (3.11) implies that

$$
\left|F^{\left(K^{0}\right)}\left(z_{K^{0}}^{*}\right)\right| \geq\left(\frac{\mathbf{L}\left(z^{0}\right)}{R^{\prime \prime}}\right)^{K^{0}}\left|F\left(z^{*}\right)\right|\left\{\frac{\left|F\left(z_{\mathbf{0}}^{*}\right)\right|}{\left|F\left(z^{*}\right)\right|}-\sum_{j=1}^{n} b_{j}\right\} .
$$

But in view of $(3.6)$ and a choice of $p_{*}$ we have

$$
\frac{\left|F\left(z_{\mathbf{0}}^{*}\right)\right|}{\left|F\left(z^{*}\right)\right|} \geq p_{*}>\sum_{j=1}^{n} b_{j}
$$

Thus, (3.7) and (3.8) imply

$$
\begin{aligned}
& \left|F^{\left(K^{0}\right)}\left(z_{K^{0}}^{*}\right)\right| \geq\left(\frac{\mathbf{L}\left(z^{0}\right)}{R^{\prime \prime}}\right)^{K^{0}}\left|F\left(z^{*}\right)\right|\left\{p_{*}-\sum_{j=1}^{n} b_{j}\right\} \\
& \geq\left(\frac{\mathbf{L}\left(z^{0}\right)}{R^{\prime \prime}}\right)^{K^{0}}\left\{p_{*}-\sum_{j=1}^{n} b_{j}\right\} \frac{\left|F^{\left(K^{0}\right)}\left(z^{0}\right)\right|\left(R^{\prime}\right)^{K^{0}}}{K^{0} ! \mathbf{L}^{K^{0}}\left(z^{0}\right)} \\
& \quad \geq\left(\frac{r_{1}^{\prime} \ldots r_{n}^{\prime}}{r_{1}^{\prime \prime} \ldots r_{n}^{\prime \prime}}\right)^{N}\left\{p_{*}-\sum_{j=1}^{n} b_{j}\right\} \frac{\left|F^{\left(K^{0}\right)}\left(z_{K^{0}}^{*}\right)\right|}{p_{0}(n !)^{n}} .
\end{aligned}
$$

Hence, we have $p_{*} \leq p_{0}\left(\frac{r_{1}^{\prime} \ldots r_{n}^{\prime}}{r_{1}^{\prime \prime} \ldots r_{n}^{\prime \prime}}\right)^{N}(N !)^{n}+\sum_{j=1}^{n} b_{j}$, but this contradicts the choice of $p_{*}$.

\section{Analogue of Theorem of Hayman for analytic in a ball function of bounded L-index in joint variables}

Theorem 4.1. Let $\mathbf{L} \in Q\left(\mathbb{B}^{n}\right)$. An analytic function $F$ in $\mathbb{B}^{n}$ has bounded $\mathbf{L}$-index in joint variables if and only if there exist $p \in \mathbb{Z}_{+}$and $c \in \mathbb{R}_{+}$such that for each $z \in \mathbb{B}^{n}$

$$
\max \left\{\frac{\left|F^{(J)}(z)\right|}{\mathbf{L}^{J}(z)}:\|J\|=p+1\right\} \leq c \cdot \max \left\{\frac{\left|F^{(K)}(z)\right|}{\mathbf{L}^{K}(z)}:\|K\| \leq p\right\} .
$$

Proof. Let $N=N\left(F, \mathbf{L}, \mathbb{B}^{n}\right)<+\infty$. The definition of the boundedness of $\mathbf{L}$-index in joint variables yields the necessity with $p=N$ and $c=((N+1) !)^{n}$.

We prove the sufficiency. For $F \equiv 0$ theorem is obvious. Thus, we suppose that $F \not \equiv 0$. Denote $\boldsymbol{\beta}=\left(\frac{\beta}{\sqrt{n}}, \ldots, \frac{\beta}{\sqrt{n}}\right)$. 
Assume that (4.1) holds, $z^{0} \in \mathbb{B}^{n}, z \in \mathbb{D}^{n}\left[z^{0}, \frac{\boldsymbol{\beta}}{\mathbf{L}\left(z^{0}\right)}\right]$. For all $J \in \mathbb{Z}_{+}^{n},\|J\| \leq p+1$, one has

$$
\begin{gathered}
\frac{\left|F^{(J)}(z)\right|}{\mathbf{L}^{J}\left(z^{0}\right)} \leq \Lambda_{2}^{J}(\boldsymbol{\beta}) \frac{\left|F^{(J)}(z)\right|}{\mathbf{L}^{J}(z)} \leq c \cdot \Lambda_{2}^{J}(\boldsymbol{\beta}) \max \left\{\frac{\left|F^{(K)}(z)\right|}{\mathbf{L}^{K}(z)}:\|K\| \leq p\right\} \\
\leq c \cdot \Lambda_{2}^{J}(\boldsymbol{\beta}) \max \left\{\Lambda_{1}^{-K}(2) \frac{\left|F^{(K)}(z)\right|}{\mathbf{L}^{K}\left(z^{0}\right)}:\|K\| \leq p\right\} \leq B G(z),
\end{gathered}
$$

where $B=c \cdot \max \left\{\Lambda_{2}^{K}(\boldsymbol{\beta}):\|K\|=p+1\right\} \max \left\{\Lambda_{1}^{-K}(\boldsymbol{\beta}):\|K\| \leq p\right\}$, and

$$
G(z)=\max \left\{\frac{\left|F^{(K)}(z)\right|}{\mathbf{L}^{K}\left(z^{0}\right)}:\|K\| \leq p\right\} .
$$

We choose

$$
z^{(1)}=\left(z_{1}^{(1)}, \ldots, z_{n}^{(1)}\right) \in \mathbb{T}^{n}\left(z^{0}, \frac{\mathbf{1}}{2 \beta \sqrt{n} \mathbf{L}\left(z^{0}\right)}\right)
$$

and

$$
z^{(2)}=\left(z_{1}^{(2)}, \ldots, z_{n}^{(2)}\right) \in \mathbb{T}^{n}\left(z^{0}, \frac{\boldsymbol{\beta}}{\mathbf{L}\left(z^{0}\right)}\right)
$$

such that $F\left(z^{(1)}\right) \neq 0$ and

$$
\left|F\left(z^{(2)}\right)\right|=M\left(\frac{\boldsymbol{\beta}}{\mathbf{L}\left(z^{0}\right)}, z^{0}, F\right) \neq 0 .
$$

These points exist, otherwise if $F(z) \equiv 0$ on skeleton

$$
\mathbb{T}^{n}\left(z^{0}, \frac{\mathbf{1}}{2 \beta \sqrt{n} \mathbf{L}\left(z^{0}\right)}\right) \quad \text { or } \quad \mathbb{T}^{n}\left(z^{0}, \frac{\boldsymbol{\beta}}{\mathbf{L}\left(z^{0}\right)}\right)
$$

then by the uniqueness theorem $F \equiv 0$ in $\mathbb{B}^{n}$. We connect the points $z^{(1)}$ and $z^{(2)}$ with plane

$$
\alpha=\left\{\begin{array}{r}
z_{2}=k_{2} z_{1}+c_{2} \\
z_{3}=k_{3} z_{1}+c_{3} \\
\cdots \\
z_{n}=k_{n} z_{1}+c_{n}
\end{array}\right.
$$

where

$$
k_{i}=\frac{z_{i}^{(2)}-z_{i}^{(1)}}{z_{1}^{(2)}-z_{1}^{(1)}}, \quad c_{i}=\frac{z_{i}^{(1)} z_{1}^{(2)}-z_{i}^{(2)} z_{1}^{(1)}}{z_{1}^{(2)}-z_{1}^{(1)}}, i=2, \ldots, n .
$$

It is easy to check that $z^{(1)} \in \alpha$ and $z^{(2)} \in \alpha$. Let $\widetilde{G}\left(z_{1}\right)=\left.G(z)\right|_{\alpha}$ be a restriction of the function $G$ onto $\alpha$.

For every $K \in \mathbb{Z}_{+}^{n}$ the function $\left.F^{(K)}(z)\right|_{\alpha}$ is analytic function of variable $z_{1}$ and $\tilde{G}\left(z_{1}^{(1)}\right)=\left.G\left(z^{(1)}\right)\right|_{\alpha} \neq 0$ because $F\left(z^{(1)}\right) \neq 0$. Hence, all zeros of the function $\left.F^{(K)}(z)\right|_{\alpha}$ are isolated as zeros of a function of one variable. Thus, zeros of the function $\tilde{G}\left(z_{1}\right)$ are isolated too. Therefore, we can choose piecewise analytic curve $\gamma$ onto $\alpha$ as following

$$
z=z(t)=\left(z_{1}(t), k_{2} z_{1}(t)+c_{2}, \ldots, k_{n} z_{1}(t)+c_{n}\right), t \in[0,1]
$$


which connect the points $z^{(1)}, z^{(2)}$ and such that $G(z(t)) \neq 0$ and

$$
\int_{0}^{1}\left|z_{1}^{\prime}(t)\right| d t \leq \frac{2 \beta}{\sqrt{n} l_{1}\left(z_{1}^{0}\right)}
$$

For a construction of the curve we connect $z_{1}^{(1)}$ and $z_{1}^{(2)}$ by a line

$$
z_{1}^{*}(t)=\left(z_{1}^{(2)}-z_{1}^{(1)}\right) t+z_{1}^{(1)}, t \in[0,1] .
$$

The curve $\gamma$ can cross points $z_{1}$ at which the function $\widetilde{G}\left(z_{1}\right)=0$. The number of such points $m=m\left(z^{(1)}, z^{(2)}\right)$ is finite. Let $\left(z_{1, k}^{*}\right)$ be a sequence of these points in ascending order of the value $\left|z_{1}^{(1)}-z_{1, k}^{*}\right|, k \in\{1,2, \ldots, m\}$. We choose

$$
r<\min _{1 \leq k \leq m-1}\left\{\left|z_{1, k}^{*}-z_{1, k+1}^{*}\right|,\left|z_{1,1}^{*}-z_{1}^{(1)}\right|,\left|z_{1, m}^{*}-z_{1}^{(2)}\right|, \frac{2 \beta^{2}-1}{2 \pi \sqrt{n} \beta l_{1}\left(z^{0}\right)}\right\} .
$$

Now we construct circles with centers at the points $z_{1, k}^{*}$ and corresponding radii $r_{k}^{\prime}<\frac{r}{2^{k}}$ such that $\widetilde{G}\left(z_{1}\right) \neq 0$ for all $z_{1}$ on the circles. It is possible, because $F \not \equiv 0$.

Every such circle is divided onto two semicircles by the line $z_{1}^{*}(t)$. The required piecewise-analytic curve consists with arcs of the constructed semicircles and segments of line $z_{1}^{*}(t)$, which connect the arcs in series between themselves or with the points $z_{1}^{(1)}, z_{1}^{(2)}$. The length of $z_{1}(t)$ in $\mathbb{C}$ (but not $z(t)$ in $\left.\mathbb{C}^{n} !\right)$ is lesser than

$$
\frac{\beta / \sqrt{n}}{l_{1}\left(z^{0}\right)}+\frac{1}{2 \sqrt{n} \beta l_{1}\left(z^{0}\right)}+\pi r \leq \frac{2 \beta}{\sqrt{n} l_{1}\left(z^{0}\right)} .
$$

Then

$$
\begin{gathered}
\int_{0}^{1}\left|z_{s}^{\prime}(t)\right| d t=\left|k_{s}\right| \int_{0}^{1}\left|z_{1}^{\prime}(t)\right| d t \leq \frac{\left|z_{s}^{(2)}-z_{s}^{(1)}\right|}{\left|z_{1}^{(2)}-z_{1}^{(1)}\right|} \frac{2 \beta}{\sqrt{n} l_{1}\left(z^{0}\right)} \\
\leq \frac{2 \beta^{2}+1}{2 \sqrt{n} \beta l_{s}\left(z^{0}\right)} \frac{2 \sqrt{n} \beta l_{1}\left(z^{0}\right)}{2 \beta^{2}-1} \frac{2 \beta}{\sqrt{n} l_{1}\left(z^{0}\right)} \leq \frac{2 \beta\left(2 \beta^{2}+1\right)}{\left(2 \beta^{2}-1\right) \sqrt{n} l_{s}\left(z^{0}\right)}, s \in\{2, \ldots, n\} .
\end{gathered}
$$

Hence,

$$
\int_{0}^{1} \sum_{s=1}^{n} l_{s}\left(z^{0}\right)\left|z_{s}^{\prime}(t)\right| d t \leq \frac{2 \beta\left(2 \beta^{2}+1\right) \sqrt{n}}{2 \beta^{2}-1}=S .
$$

Since the function $z=z(t)$ is piece-wise analytic on $[0,1]$, then for arbitrary $K \in \mathbb{Z}_{+}^{n}$, $J \in \mathbb{Z}_{+}^{n},\|K\| \leq p$, either

$$
\frac{\left|F^{(K)}(z(t))\right|}{\mathbf{L}^{K}\left(z^{0}\right)} \equiv \frac{|F(J)(z(t))|}{\mathbf{L}^{J}\left(z^{0}\right)}
$$

or the equality

$$
\frac{\left|F^{(K)}(z(t))\right|}{\mathbf{L}^{K}\left(z^{0}\right)}=\frac{\left|F^{(J)}(z(t))\right|}{\mathbf{L}^{J}\left(z^{0}\right)}
$$

holds only for a finite set of points $t_{k} \in[0 ; 1]$.

Then for function $G(z(t))$ as maximum of such expressions $\frac{\left|F^{(J)}(z(t))\right|}{\mathbf{L}^{J}\left(z^{0}\right)}$ by all $\|J\| \leq p$ two cases are possible: 
1. In some interval of analyticity of the curve $\gamma$ the function $G(z(t))$ identically equals simultaneously to some derivatives, that is (4.5) holds. It means that $G(z(t)) \equiv \frac{\left|F^{(J)}(z(t))\right|}{\mathbf{L}^{J}\left(z^{0}\right)}$ for some $J,\|J\| \leq p$. Clearly, the function $F^{(J)}(z(t))$ is analytic. Then $\left|F^{(J)}(z(t))\right|$ is continuously differentiable function on the interval of analyticity except points where this partial derivative equals zero $\left|F^{\left(j_{1}, j_{2}\right)}\left(z_{1}(t), z_{2}(t)\right)\right|=0$. However, there are not the points, because in the opposite case $G(z(t))=0$. But it contradicts the construction of the curve $\gamma$.

2. In some interval of analyticity of the curve $\gamma$ the function $G(z(t))$ equals simultaneously to some derivatives at a finite number of points $t_{k}$, that is (4.6) holds. Then the points $t_{k}$ divide interval of analyticity onto a finite number of segments, in which of them $G(z(t))$ equals to one from the partial derivatives, i. e. $G(z(t)) \equiv \frac{\left|F^{(J)}(z(t))\right|}{\mathbf{L}^{J}\left(z^{0}\right)}$ for some $J,\|J\| \leq p$. As above, in each from these segments the functions $\left|F^{(J)}(z(t))\right|$, and $G(z(t))$ are continuously differentiable except the points $t_{k}$.

The inequality

$$
\frac{d}{d t}|f(t)| \leq\left|\frac{d f(t)}{d t}\right|
$$

holds for complex-valued functions of real argument outside a countable set of points. In view of this fact and (4.2) we have

$$
\begin{gathered}
\frac{d}{d t} G(z(t)) \leq \max \left\{\frac{1}{\mathbf{L}^{J}\left(z^{0}\right)}\left|\frac{d}{d t} F^{(J)}(z(t))\right|:\|J\| \leq p\right\} \\
\leq \max \left\{\sum_{s=1}^{n}\left|\frac{\partial^{\|J\|+1} F}{\partial z_{1}^{j_{1}} \ldots \partial z_{s}^{j_{s}+1} \ldots \partial z_{n}^{j_{n}}}(z(t))\right| \frac{\left|z_{s}^{\prime}(t)\right|}{\mathbf{L}^{j}\left(z^{0}\right)}:\|J\| \leq p\right\} \\
\leq \max \left\{\sum_{s=1}^{n}\left|\frac{\partial^{\|J\|+1} F}{\partial z_{1}^{j_{1}} \ldots \partial z_{s}^{j_{s}+1} \ldots \partial z_{n}^{j_{n}}}(z(t))\right| \frac{\left.l^{0}\right)\left|z_{s}^{\prime}(t)\right|}{l_{1}^{j_{1}}\left(z^{0}\right) \ldots l_{s}^{j_{1}+1}\left(z^{0}\right) \ldots l_{n}^{j_{n}}\left(z^{0}\right)}:\right. \\
\|J\| \leq p\} \leq\left(\sum_{s=1}^{n} l_{s}\left(z^{0}\right)\left|z_{s}^{\prime}(t)\right|\right) \max \left\{\frac{\left|F^{(j)}(z(t))\right|}{\mathbf{L}^{J}\left(z^{0}\right)}:\|J\| \leq p+1\right\} \\
\leq\left(\sum_{s=1}^{n} l_{s}\left(z^{0}\right)\left|z_{s}^{\prime}(t)\right|\right) B G(z(t)) .
\end{gathered}
$$

Therefore, (4.4) yields

$$
\left|\ln \frac{G\left(z^{(2)}\right)}{G\left(z^{(1)}\right)}\right|=\left|\int_{0}^{1} \frac{1}{G(z(t))} \frac{d}{d t} G(z(t)) d t\right| \leq B \int_{0}^{1} \sum_{s=1}^{n} l_{s}\left(z^{0}\right)\left|z_{s}^{\prime}(t)\right| d t \leq S \cdot B .
$$

Using (4.3), we deduce

$$
M\left(\frac{\boldsymbol{\beta}}{\mathbf{L}\left(z^{0}\right)}, z^{0}, F\right) \leq G\left(z^{(2)}\right) \leq G\left(z^{(1)}\right) e^{S B} .
$$


Since $z^{(1)} \in \mathbb{T}^{n}\left(z^{0}, \frac{1}{2 \beta \sqrt{n} \mathbf{L}\left(z^{0}\right)}\right)$, the Cauchy inequality holds

$$
\frac{\left|F^{(J)}\left(z^{(1)}\right)\right|}{\mathbf{L}^{J}\left(z^{0}\right)} \leq J !(2 \beta \sqrt{n})^{\|J\|} M\left(\frac{\mathbf{1}}{2 \beta \sqrt{n} \mathbf{L}\left(z^{0}\right)}, z^{0}, F\right) .
$$

for all $J \in \mathbb{Z}_{+}^{n}$. Therefore, for $\|J\| \leq p$ we obtain

$$
\begin{gathered}
G\left(z^{(1)}\right) \leq(p !)^{n}(2 \beta \sqrt{n})^{p} M\left(\frac{\mathbf{1}}{2 \beta \sqrt{n} \mathbf{L}\left(z^{0}\right)}, z^{0}, F\right), \\
M\left(\frac{\boldsymbol{\beta}}{\mathbf{L}\left(z^{0}\right)}, z^{0}, F\right) \leq e^{S B}(p !)^{n}(2 \beta \sqrt{n})^{p} M\left(\frac{\mathbf{1}}{2 \beta \sqrt{n} \mathbf{L}\left(z^{0}\right)}, z^{0}, F\right) .
\end{gathered}
$$

Hence, by Theorem 3.1 the function $F$ has bounded $\mathbf{L}$-index in joint variables.

The following result was also obtained for other classes of holomorphic functions in $[21,11,7]$.

Theorem 4.2. Let $\mathbf{L} \in Q\left(\mathbb{B}^{n}\right)$. An analytic function $F$ in $\mathbb{B}^{n}$ has bounded $\mathbf{L}$-index in joint variables if and only if there exist $c \in(0 ;+\infty)$ and $N \in \mathbb{N}$ such that for each $z \in \mathbb{B}^{n}$ the inequality

$$
\sum_{\|K\|=0}^{N} \frac{\left|F^{(K)}(z)\right|}{K ! \mathbf{L}^{K}(z)} \geq c \sum_{\|K\|=N+1}^{\infty} \frac{\left|F^{(K)}(z)\right|}{K ! \mathbf{L}^{K}(z)}
$$

Proof. Let $\frac{1}{\beta}<\theta_{j}<1, j \in\{1, \ldots, n\}, \Theta=\left(\theta_{1}, \ldots, \theta_{n}\right)$. If the function $F$ has bounded L-index in joint variables then by Theorem $2.2 F$ has bounded $\widetilde{\mathbf{L}}$-index in joint variables, where $\widetilde{\mathbf{L}}=\left(\widetilde{l}_{1}(z), \ldots, \widetilde{l}_{n}(z)\right), \widetilde{l}_{j}(z)=\theta_{j} l_{j}(z), j \in\{1, \ldots, n\}$. Let $\widetilde{N}=N\left(F, \widetilde{L}, \mathbb{B}^{n}\right)$. Therefore,

$$
\begin{gathered}
\max \left\{\frac{\left|F^{(K)}(z)\right|}{K ! \mathbf{L}^{K}(z)}:\|K\| \leq \widetilde{N}\right\}=\max \left\{\frac{\Theta^{K}\left|F^{(K)}(z)\right|}{K ! \widetilde{\mathbf{L}}^{K}(z)}:\|K\| \leq \widetilde{N}\right\} \\
\geq \prod_{s=1}^{n} \theta_{s}^{\widetilde{N}} \max \left\{\frac{\left|F^{(K)}(z)\right|}{K ! \widetilde{\mathbf{L}}^{K}(z)}:\|K\| \leq \widetilde{N}\right\} \geq \prod_{s=1}^{n} \theta_{s}^{\widetilde{N}} \frac{\left|F^{(J)}(z)\right|}{J ! \widetilde{\mathbf{L}}^{J}(z)}=\prod_{s=1}^{n} \theta_{s}^{\widetilde{N}-j_{s}} \frac{\left|F^{(J)}(z)\right|}{J ! \mathbf{L}^{J}(z)}
\end{gathered}
$$

for all $J \geq \mathbf{0}$ and

$$
\begin{aligned}
& \sum_{\|J\|=\widetilde{N}+1}^{\infty} \frac{\left|F^{(J)}(z)\right|}{J ! \mathbf{L}^{j}(z)} \leq \max \left\{\frac{\left|F^{(K)}(z)\right|}{K ! \mathbf{L}^{K}(z)}:\|K\| \leq \widetilde{N}\right\} \sum_{\|J\|=\widetilde{N}+1}^{\infty} \theta_{s}^{j_{s}-\widetilde{N}} \\
= & \prod_{i=1}^{n} \frac{\theta_{s}}{1-\theta_{s}} \max \left\{\frac{\left|F^{(K)}(z)\right|}{K ! \mathbf{L}^{K}(z)}:\|K\| \leq \widetilde{N}\right\} \leq \prod_{i=1}^{n} \frac{\theta_{s}}{1-\theta_{s}} \sum_{\|K\|=0}^{\tilde{N}} \frac{\left|F^{(K)}(z)\right|}{K ! \mathbf{L}^{K}(z)} .
\end{aligned}
$$

Hence, we obtain (4.7) with $N=\widetilde{N}$ and

$$
c=\prod_{i=1}^{n} \frac{\theta_{s}}{1-\theta_{s}} \text {. }
$$


On the contrary, inequality (4.7) implies

$$
\begin{gathered}
\max \left\{\frac{\left|F^{(J)}(z)\right|}{J ! \mathbf{L}^{J}(z)}:\|J\|=N+1\right\} \leq \sum_{\|K\|=N+1}^{\infty} \frac{\left|F^{(K)}(z)\right|}{K ! \mathbf{L}^{K}(z)} \leq \frac{1}{c} \sum_{\|K\|=0}^{N} \frac{\left|F^{(K)}(z)\right|}{K ! \mathbf{L}^{K}(z)} \\
\leq \frac{1}{c} \sum_{i=0}^{N} C_{n+i-1}^{i} \max \left\{\frac{\left|F^{(K)}(z)\right|}{K ! \mathbf{L}^{K}(z)}:\|K\| \leq N\right\}
\end{gathered}
$$

and by Theorem $4.1 F$ is of bounded $\mathbf{L}$-index in joint variables.

\section{Some application for PDE: a scheme}

Here we present a scheme of application of Hayman's Theorem to PDE. This is also appilcable in a more general situation.

Let us consider the following system of partial differential equations:

$$
\left\{\begin{array}{l}
F^{(2,0)}\left(z_{1}, z_{2}\right)=2 \pi z_{2} \tan \left(\pi z_{1} z_{2}\right) F^{(1,0)}\left(z_{1}, z_{2}\right) \\
F^{(0,2)}\left(z_{1}, z_{2}\right)=2 \pi z_{1} \tan \left(\pi z_{1} z_{2}\right) F^{(0,1)}\left(z_{1}, z_{2}\right)
\end{array}\right.
$$

Differentiate in variables $z_{1}$ and $z_{2}$ we deduce

$$
\left\{\begin{aligned}
F^{(3,0)}\left(z_{1}, z_{2}\right) & =\frac{2 \pi^{2} z_{2}^{2}}{\cos ^{2}\left(\pi z_{1} z_{2}\right)} F^{(1,0)}\left(z_{1}, z_{2}\right)+2 \pi z_{2} \tan \left(\pi z_{1} z_{2}\right) F^{(2,0)}\left(z_{1}, z_{2}\right), \\
F^{(2,1)}\left(z_{1}, z_{2}\right) & =2 \pi \tan \left(\pi z_{1} z_{2}\right) F^{(1,0)}\left(z_{1}, z_{2}\right)+\frac{2 \pi^{2} z_{1} z_{2}}{\cos ^{2}\left(\pi z_{1} z_{2}\right)} F^{(1,0)}\left(z_{1}, z_{2}\right)+ \\
& +2 \pi z_{2} \tan \left(\pi z_{1} z_{2}\right) F^{(1,1)}\left(z_{1}, z_{2}\right), \\
& +2 \pi z_{1} \tan \left(\pi z_{1} z_{2}\right) F^{(1,1)}\left(z_{1}, z_{2}\right), \\
F^{(1,2)}\left(z_{1}, z_{2}\right) & =2 \pi \tan \left(\pi z_{1} z_{2}\right) F^{(0,1)}\left(z_{1}, z_{2}\right)+\frac{2 \pi^{2} z_{1} z_{2}}{\cos ^{2}\left(\pi z_{1} z_{2}\right)} F^{(1,0)}\left(z_{1}, z_{2}\right)+ \\
F^{(0,3)}\left(z_{1}, z_{2}\right) & =\frac{2 \pi^{2} z_{1}^{2}}{\cos ^{2}\left(\pi z_{1} z_{2}\right)} F^{(1,0)}\left(z_{1}, z_{2}\right)+2 \pi z_{1} \tan \left(\pi z_{1} z_{2}\right) F^{(2,0)}\left(z_{1}, z_{2}\right),
\end{aligned}\right.
$$

Let

$$
\mathbf{L}\left(z_{1}, z_{2}\right)=\left(l_{1}\left(z_{1}, z_{2}\right), l_{2}\left(z_{1}, z_{2}\right)\right)=\left(\frac{\left|z_{2}\right|+1}{(1-|z|)\left|\frac{1}{2}-z_{1} z_{2}\right|}, \frac{\left|z_{1}\right|+1}{(1-|z|)\left|\frac{1}{2}-z_{1} z_{2}\right|}\right),
$$

where $z=\left(z_{1}, z_{2}\right),|z|=\sqrt{\left|z_{1}\right|^{2}+\left|z_{2}\right|^{2}}$. Now we will estimate all third order partial derivatives of the function $F\left(z_{1}, z_{2}\right)$ by its first and second order partial derivatives. From the first equation of system (5.1) we have for all $z \in \mathbb{B}^{2}$ :

$$
\begin{aligned}
& \frac{\left|F^{(3,0)}\left(z_{1}, z_{2}\right)\right|}{l_{1}^{3}\left(z_{1}, z_{2}\right)} \leq \frac{2 \pi^{2}\left|z_{2}\right|^{2}\left|F^{(1,0)}\left(z_{1}, z_{2}\right)\right|}{\left|\cos ^{2}\left(\pi z_{1} z_{2}\right)\right| l_{1}^{3}\left(z_{1}, z_{2}\right)}+2 \pi\left|z_{2} \tan \left(\pi z_{1} z_{2}\right)\right| \frac{\left|F^{(2,0)}\left(z_{1}, z_{2}\right)\right|}{l_{1}^{3}\left(z_{1}, z_{2}\right)} \\
& \leq\left(\frac{2 \pi^{2}\left|z_{2}\right|^{2}}{\left|\cos ^{2}\left(\pi z_{1} z_{2}\right)\right| l_{1}^{2}\left(z_{1}, z_{2}\right)}+\frac{2 \pi\left|z_{2} \tan \left(\pi z_{1} z_{2}\right)\right|}{l_{1}\left(z_{1}, z_{2}\right)}\right) \max _{j \in\{1,2\}}\left\{\frac{\left|F^{(j, 0)}\left(z_{1}, z_{2}\right)\right|}{l_{1}^{j}\left(z_{1}, z_{2}\right)}\right\} \\
& \quad \leq\left(2 \pi^{2} \frac{(1-|z|)^{2}\left|\frac{1}{2}-z_{1} z_{2}\right|^{2}}{\left|\cos ^{2}\left(\pi z_{1} z_{2}\right)\right|}+2 \pi\left|\tan \left(\pi z_{1} z_{2}\right)\right|(1-|z|)\left|\frac{1}{2}-z_{1} z_{2}\right|\right)
\end{aligned}
$$




$$
\begin{gathered}
\times \max \left\{\frac{\left|F^{(j, 0)}\left(z_{1}, z_{2}\right)\right|}{l_{1}^{j}\left(z_{1}, z_{2}\right)}: j \in\{1,2\}\right\} \\
=\left(\frac{(1-|z|)^{2}\left|\frac{\pi}{2}-\pi z_{1} z_{2}\right|^{2}}{\left|\sin ^{2}\left(\frac{\pi}{2}-\pi z_{1} z_{2}\right)\right|}+2\left|\sin \left(\pi z_{1} z_{2}\right)\right|(1-|z|) \frac{\left|\frac{\pi}{2}-\pi z_{1} z_{2}\right|}{\left|\sin \left(\frac{\pi}{2}-\pi z_{1} z_{2}\right)\right|}\right) \\
\times \max \left\{\frac{\left|F^{(j, 0)}\left(z_{1}, z_{2}\right)\right|}{l_{1}^{j}\left(z_{1}, z_{2}\right)}: j \in\{1,2\}\right\} \leq C \max \left\{\frac{\left|F^{(j, 0)}\left(z_{1}, z_{2}\right)\right|}{l_{1}^{j}\left(z_{1}, z_{2}\right)}: j \in\{1,2\}\right\} .
\end{gathered}
$$

Similarly, the second equation of system (5.1) yields

$$
\begin{aligned}
& \frac{\left|F^{(2,1)}\left(z_{1}, z_{2}\right)\right|}{l_{1}^{2}\left(z_{1}, z_{2}\right) l_{2}\left(z_{1}, z_{2}\right)} \leq\left(\frac{2 \pi\left|\tan \left(\pi z_{1} z_{2}\right)\right|}{l_{1}\left(z_{1}, z_{2}\right) l_{2}\left(z_{1}, z_{2}\right)}+\frac{2 \pi^{2}\left|z_{1} z_{2}\right|}{\left|\cos ^{2}\left(\pi z_{1} z_{2}\right)\right| l_{1}\left(z_{1}, z_{2}\right) l_{2}\left(z_{1}, z_{2}\right)}\right) \\
& \times \frac{\left|F^{(1,0)}\left(z_{1}, z_{2}\right)\right|}{l_{1}\left(z_{1}, z_{2}\right)}+\frac{2 \pi\left|z_{2} \tan \left(\pi z_{1} z_{2}\right)\right|}{l_{1}\left(z_{1}, z_{2}\right)} \frac{\left|F^{(1,1)}\left(z_{1}, z_{2}\right)\right|}{l_{1}\left(z_{1}, z_{2}\right) l_{2}\left(z_{1}, z_{2}\right)} \\
& \leq\left(\frac{2 \pi\left|\sin \left(\pi z_{1} z_{2}\right)\right|(1-|z|)^{2}\left|\frac{1}{2}-z_{1} z_{2}\right|^{2}}{\left|\cos \left(\pi z_{1} z_{2}\right)\right|}\right. \\
& \left.+\frac{2 \pi^{2}(1-|z|)^{2}\left|\frac{1}{2}-z_{1} z_{2}\right|^{2}}{\left|\cos ^{2}\left(\pi z_{1} z_{2}\right)\right|}+\frac{2 \pi\left|\sin \left(\pi z_{1} z_{2}\right)\right|(1-|z|)\left|\frac{1}{2}-z_{1} z_{2}\right|}{\left|\cos \left(\pi z_{1} z_{2}\right)\right|}\right) \\
& \times \max \left\{\frac{\left|F^{(1, j)}\left(z_{1}, z_{2}\right)\right|}{l_{1}\left(z_{1}, z_{2}\right) l_{2}^{j}\left(z_{1}, z_{2}\right)}: j \in\{0,1\}\right\} \\
& \leq\left(\frac{2 \pi\left|\sin \left(\pi z_{1} z_{2}\right)\right|(1-|z|)^{2}\left|\frac{1}{2}-z_{1} z_{2}\right|^{2}}{\left|\sin \left(\frac{\pi}{2}-\pi z_{1} z_{2}\right)\right|}+\frac{2(1-|z|)^{2}\left|\frac{\pi}{2}-\pi z_{1} z_{2}\right|^{2}}{\left|\sin ^{2}\left(\frac{\pi}{2}-\pi z_{1} z_{2}\right)\right|}\right. \\
& \left.+\frac{2\left|\sin \left(\pi z_{1} z_{2}\right)\right|(1-|z|)\left|\frac{\pi}{2}-\pi z_{1} z_{2}\right|}{\left|\sin \left(\frac{\pi}{2}-\pi z_{1} z_{2}\right)\right|}\right) \max \left\{\frac{\left|F^{(1, j)}\left(z_{1}, z_{2}\right)\right|}{l_{1}\left(z_{1}, z_{2}\right) l_{2}^{j}\left(z_{1}, z_{2}\right)}: j \in\{0,1\}\right\} \\
& \leq C \max \left\{\frac{\left|F^{(1, j)}\left(z_{1}, z_{2}\right)\right|}{l_{1}\left(z_{1}, z_{2}\right) l_{2}^{j}\left(z_{1}, z_{2}\right)}: j \in\{0,1\}\right\} \text {. }
\end{aligned}
$$

By analogy, we can prove similar estimates for the third and the fourth equation of system (5.1). Combining all estimates, one has

$$
\begin{aligned}
& \max \left\{\frac{\left|F^{(k, 3-k)}\left(z_{1}, z_{2}\right)\right|}{l_{1}^{k}\left(z_{1}, z_{2}\right) l_{2}^{3-k}\left(z_{1}, z_{2}\right)}: k \in\{0,1,2,3\}\right\} \\
\leq & C \max \left\{\frac{\left|F^{(k, j)}\left(z_{1}, z_{2}\right)\right|}{l_{1}^{k}\left(z_{1}, z_{2}\right) l_{2}^{j}\left(z_{1}, z_{2}\right)}: 0 \leq k+j \leq 2\right\} .
\end{aligned}
$$

Hence, by Theorem 4.1 every analytic solution in $\mathbb{B}^{2}$ of system (5.1) has bounded L-index in joint variables with

$$
\mathbf{L}\left(z_{1}, z_{2}\right)=\left(\frac{\left|z_{2}\right|+1}{(1-|z|)\left|\frac{1}{2}-z_{1} z_{2}\right|}, \frac{\left|z_{1}\right|+1}{(1-|z|)\left|\frac{1}{2}-z_{1} z_{2}\right|}\right) .
$$


Particularly, the function $F\left(z_{1}, z_{2}\right)=\tan \left(\pi z_{1} z_{2}\right)$ has the bounded $\mathbf{L}$-index in joint variables. Indeed, it is easy to see that the function $F$ is analytic solution in $\mathbb{B}^{2}$ of system (5.1).

\section{Boundedness of $l_{j}$-index in every direction $\mathbf{1}_{j}$}

This section shows another application of Theorem 3.1. The boundedness of $l_{j}$ index of a function $F$ in every variable $z_{j}$, generally speaking, does not imply the boundedness of $\mathbf{L}$-index in joint variables (see example in [4]). But, if $F$ has bounded $l_{j}$-index in every direction $\mathbf{1}_{j}, j \in\{1, \ldots, n\}$, then $F$ is a function of bounded $\mathbf{L}$-index in joint variables.

Let $\mathbf{b}=\left(b_{1}, \ldots, b_{n}\right) \in \mathbb{C}^{n} \backslash\{\mathbf{0}\}$ be a given direction, $L: \mathbb{B}^{n} \rightarrow \mathbb{R}_{+}$be a continuous function such that for all $z \in \mathbb{B}^{n} L(z)>\frac{\beta|\mathbf{b}|}{1-|z|}, \beta>1$.

For $\eta \in[0, \beta], z \in \mathbb{B}^{n}$, we define

$$
\begin{aligned}
& \lambda_{1}^{\mathbf{b}}(z, \eta, L)=\inf \left\{L(z+t \mathbf{b}) / L(z):|t| \leq \frac{\eta}{L(z)}\right\}, \\
& \lambda_{1}^{\mathbf{b}}(\eta, L)=\inf \left\{\lambda_{1}^{\mathbf{b}}(z, \eta, L): z \in \mathbb{B}^{n}\right\}, \\
& \lambda_{2}^{\mathbf{b}}(z, \eta, L)=\sup \left\{L(z+t \mathbf{b}) / L(z):|t| \leq \frac{\eta}{L(z)}\right\}, \\
& \lambda_{2}^{\mathbf{b}}(\eta, L)=\sup \left\{\lambda_{2}^{\mathbf{b}}(z, \eta, L): z \in \mathbb{B}^{n}\right\} .
\end{aligned}
$$

By $Q_{\mathbf{b}, \beta}\left(\mathbb{B}^{n}\right)$ we denote the class of all functions $L$ satisfying $\forall \eta \in[0, \beta]$,

$$
0<\lambda_{1}^{\mathbf{b}}(\eta, L) \leq \lambda_{2}^{\mathbf{b}}(\eta, L)<+\infty .
$$

Analytic in $\mathbb{B}^{n}$ function $F(z)$ is called a function of bounded L-index in the direction $\mathbf{b}$, if there exists $m_{0} \in \mathbb{Z}_{+}$that for every $m \in \mathbb{Z}_{+}$and for every $z \in \mathbb{B}^{n}$ the following inequality is valid

$$
\frac{1}{m ! L^{m}(z)}\left|\frac{\partial^{m} F(z)}{\partial \mathbf{b}^{m}}\right| \leq \max \left\{\frac{1}{k ! L^{k}(z)}\left|\frac{\partial^{k} F(z)}{\partial \mathbf{b}^{k}}\right|: 0 \leq k \leq m_{0}\right\},
$$

where

$$
\left.\frac{\partial^{0} F(z)}{\partial \mathbf{b}^{0}}=F(z), \frac{\partial F(z)}{\partial \mathbf{b}}=\sum_{j=1}^{n} \frac{\partial F(z)}{\partial z_{j}} b_{j}, \overline{\mathbf{b}}\right\rangle, \frac{\partial^{k} F(z)}{\partial \mathbf{b}^{k}}=\frac{\partial}{\partial \mathbf{b}}\left(\frac{\partial^{k-1} F(z)}{\partial \mathbf{b}^{k-1}}\right), k \geq 2 .
$$

The least such integer $m_{0}$ is called the $L$-index in the direction $\mathbf{b}$ of the analytic function $F$ and is denoted by $N_{\mathbf{b}}(F, L)=m_{0}$. In the case $n=1, \mathbf{b}=1$ and $L=l$ we obtain a definition of analytic in an unit disc function of bounded $l$-index $[22,21]$.

We need the following theorem.

Theorem 6.1 ([3]). Let $\beta>1, L \in Q_{\mathbf{b}, \beta}\left(\mathbb{B}^{n}\right)$. Analytic in $\mathbb{B}^{n}$ function $F(z)$ is of bounded L-index in the direction $\mathbf{b} \in \mathbb{C}^{n}$ if and only if for any $r_{1}$ and any $r_{2}$ with $0<r_{1}<r_{2} \leq \beta$, there exists number $P_{1}=P_{1}\left(r_{1}, r_{2}\right) \geq 1$ such that for each $z^{0} \in \mathbb{B}^{n}$

$$
\max \left\{\left|F\left(z^{0}+t \mathbf{b}\right)\right|:|t|=\frac{r_{2}}{L\left(z^{0}\right)}\right\} \leq P_{1} \max \left\{\left|F\left(z^{0}+t \mathbf{b}\right)\right|:|t|=\frac{r_{1}}{L\left(z_{0}\right)}\right\} .
$$

It is easy to see that if $\mathbf{L}(z)=\left(l_{1}(z), \ldots, l_{n}(z)\right)$ and $\mathbf{L} \in Q\left(\mathbb{B}^{n}\right)$, then

$$
l_{j} \in Q_{\mathbf{1}_{j}, \beta / \sqrt{n}}\left(\mathbb{B}^{n}\right), j \in\{1, \ldots, n\} .
$$


Theorem 6.2. Let $\mathbf{L}(z)=\left(l_{1}(z), \ldots, l_{n}(z)\right), \mathbf{L} \in Q\left(\mathbb{B}^{n}\right)$. If an analytic in $\mathbb{B}^{n}$ function $F$ has bounded $l_{j}$-index in the direction $\mathbf{1}_{j}$ for every $j \in\{1, \ldots, n\}$, then $F$ is of bounded $\mathbf{L}$-index in joint variables.

Proof. Let $F$ be an analytic in $\mathbb{B}^{n}$ function of bounded $l_{j}$-index in every direction $\mathbf{1}_{j}$. Then by Theorem 6.1 for every $j \in\{1, \ldots, n\}$ and arbitrary $0<r_{j}^{\prime}<1<r_{j}^{\prime \prime} \leq \frac{\beta}{\sqrt{n}}$ there exists a number $p_{j}=p_{j}\left(r^{\prime}, r^{\prime \prime}\right)$ such that for every $\left(z_{1}, \ldots, z_{j-1}, z_{j}^{0}, z_{j+1}, \ldots, z_{n}\right) \in \mathbb{B}^{n}$,

$$
\begin{aligned}
\max & \left\{|F(z)|:\left|z_{j}-z_{j}^{0}\right|=\frac{r_{j}^{\prime \prime}}{l_{j}\left(z_{1}, \ldots, z_{j-1}, z_{j}^{0}, z_{j+1}, \ldots, z_{n}\right)}\right\} \leq p_{j}\left(r_{j}^{\prime}, r_{j}^{\prime \prime}\right) \\
& \times \max \left\{|F(z)|:\left|z_{j}-z_{j}^{0}\right|=\frac{r_{j}^{\prime}}{l_{j}\left(z_{1}, \ldots, z_{j-1}, z_{j}^{0}, z_{j+1}, \ldots, z_{n}\right)}\right\} .
\end{aligned}
$$

Obviously, if for every $j \in\{1, \ldots, n\} \quad l_{j} \in Q_{\mathbf{1}_{j}, \beta / \sqrt{n}}\left(\mathbb{B}^{n}\right)$ then $\mathbf{L} \in Q\left(\mathbb{B}^{n}\right)$. Let $z^{0}$ be an arbitrary point in $\mathbb{B}^{n}$, and a point $z^{*} \in \mathbb{T}^{n}\left(z^{0}, \frac{R^{\prime \prime}}{\mathbf{L}\left(z^{0}\right)}\right)$ is such that

$$
M\left(\frac{R^{\prime \prime}}{\mathbf{L}\left(z^{0}\right)}, z^{0}, F\right)=\left|F\left(z^{*}\right)\right| .
$$

We choose $R^{\prime \prime}$ and $R^{\prime}$ such that $1<R^{\prime \prime} \leq\left(\frac{\beta}{\sqrt{n}}, \ldots, \frac{\beta}{\sqrt{n}}\right)$ and $R^{\prime}<\Lambda_{1}\left(R^{\prime \prime}\right)$. Then inequality (6.3) implies that

$$
\begin{aligned}
& M\left(\frac{R^{\prime \prime}}{\mathbf{L}\left(z^{0}\right)}, z^{0}, F\right) \leq \max \left\{\left|F\left(z_{1}, z_{2}^{*}, z_{3}^{*}, \ldots, z_{n}^{*}\right)\right|:\left|z_{1}-z_{1}^{0}\right|=\frac{r_{1}^{\prime \prime}}{l_{1}\left(z^{0}\right)}\right\} \\
& =\max \left\{\left|F\left(z_{1}, z_{2}^{*}, \ldots, z_{n}^{*}\right)\right|:\left|z_{1}-z_{1}^{0}\right|=\frac{r_{1}^{\prime \prime}}{l_{1}\left(z_{1}^{0}, z_{2}^{*}, \ldots, z_{n}^{*}\right)} \frac{l_{1}\left(z_{1}^{0}, z_{2}^{*}, \ldots, z_{n}^{*}\right)}{l_{1}\left(z^{0}\right)}\right\} \\
& \leq \max \left\{\left|F\left(z_{1}, z_{2}^{*}, \ldots, z_{n}^{*}\right)\right|:\left|z_{1}-z_{1}^{0}\right|=\frac{r_{1}^{\prime \prime} \lambda_{2,1}\left(R^{\prime \prime}\right)}{l_{1}\left(z_{1}^{0}, z_{2}^{*}, \ldots, z_{n}^{*}\right)}\right\} \\
& \leq p_{1}\left(r_{1}^{\prime}, r_{1}^{\prime \prime} \lambda_{2,1}\left(R^{\prime \prime}\right)\right) \max \left\{\left|F\left(z_{1}, z_{2}^{*}, \ldots, z_{n}^{*}\right)\right|:\left|z_{1}-z_{1}^{0}\right|=\frac{r_{1}^{\prime}}{l_{1}\left(z_{1}^{0}, z_{2}^{*}, \ldots, z_{n}^{*}\right)}\right\} \\
& =p_{1}\left(r_{1}^{\prime}, r_{1}^{\prime \prime} \lambda_{2,1}\left(R^{\prime \prime}\right)\right) \\
& \times \max \left\{\left|F\left(z_{1}, z_{2}^{*}, \ldots, z_{n}^{*}\right)\right|:\left|z_{1}-z_{1}^{0}\right|=\frac{r_{1}^{\prime}}{l_{1}\left(z^{0}\right)} \frac{l_{1}\left(z^{0}\right)}{l_{1}\left(z_{1}^{0}, z_{2}^{*}, \ldots, z_{n}^{*}\right)}\right\} \\
& \leq p_{1}\left(r_{1}^{\prime}, r_{1}^{\prime \prime} \lambda_{2,1}\left(R^{\prime \prime}\right)\right) \max \left\{\left|F\left(z_{1}, z_{2}^{*}, \ldots, z_{n}^{*}\right)\right|:\left|z_{1}-z_{1}^{0}\right|=\frac{r_{1}^{\prime}}{\lambda_{1,1}\left(R^{\prime \prime}\right) l_{1}\left(z^{0}\right)}\right\} \\
& =p_{1}\left(r_{1}^{\prime}, r_{1}^{\prime \prime} \lambda_{2,1}\left(R^{\prime \prime}\right)\right)\left|F\left(z_{1}^{* *}, z_{2}^{*}, \ldots, z_{n}^{*}\right)\right| \leq p_{1}\left(r_{1}^{\prime}, r_{1}^{\prime \prime} \lambda_{2,1}\left(R^{\prime \prime}\right)\right) \\
& \times \max \left\{\left|F\left(z_{1}^{* *}, z_{2}, z_{3}^{*}, \ldots, z_{n}^{*}\right)\right|:\left|z_{2}-z_{2}^{0}\right|=\frac{r_{2}^{\prime \prime}}{l_{2}\left(z^{0}\right)}\right\}=p_{1}\left(r_{1}^{\prime}, r_{1}^{\prime \prime} \lambda_{2,1}\left(R^{\prime \prime}\right)\right) \\
& \times \max \left\{\left|F\left(z_{1}^{* *}, z_{2}, \ldots, z_{n}^{*}\right)\right|:\left|z_{2}-z_{2}^{0}\right|=\frac{r_{2}^{\prime \prime}}{l_{2}\left(z_{1}^{* *}, z_{2}^{0}, \ldots, z_{n}^{*}\right)} \frac{l_{2}\left(z_{1}^{* *}, z_{2}^{0}, \ldots, z_{n}^{*}\right)}{l_{2}\left(z^{0}\right)}\right\} \\
& \leq p_{1}\left(r_{1}^{\prime}, r_{1}^{\prime \prime} \lambda_{2,1}\left(R^{\prime \prime}\right)\right) \max \left\{\left|F\left(z_{1}^{* *}, z_{2}, \ldots, z_{n}^{*}\right)\right|:\left|z_{2}-z_{2}^{0}\right|=\frac{r_{2}^{\prime \prime} \lambda_{2,2}\left(R^{\prime \prime}\right)}{l_{2}\left(z_{1}^{* *}, z_{2}^{0}, \ldots, z_{n}^{*}\right)}\right\}
\end{aligned}
$$




$$
\begin{gathered}
\leq \prod_{j=1}^{2} p_{j}\left(r_{j}^{\prime}, r_{j}^{\prime \prime} \lambda_{2, j}\left(R^{\prime \prime}\right)\right) \\
\quad \times \max \left\{\left|F\left(z_{1}^{* *}, z_{2}, \ldots, z_{n}^{*}\right)\right|:\left|z_{2}-z_{2}^{0}\right|=\frac{r_{2}^{\prime}}{l_{2}\left(z_{1}^{* *}, z_{2}^{0}, \ldots, z_{n}^{*}\right)}\right\} \\
\leq \prod_{j=1}^{2} p_{j}\left(r_{j}^{\prime}, r_{j}^{\prime \prime} \lambda_{2, j}\left(R^{\prime \prime}\right)\right) \max \left\{\left|F\left(z_{1}^{* *}, z_{2}, \ldots, z_{n}^{*}\right)\right|:\left|z_{2}-z_{2}^{0}\right|=\frac{r_{2}^{\prime}}{\lambda_{1,2}\left(R^{\prime \prime}\right) l_{2}\left(z^{0}\right)}\right\} \\
=\prod_{j=1}^{2} p_{j}\left(r_{j}^{\prime}, r_{j}^{\prime \prime} \lambda_{2, j}\left(R^{\prime \prime}\right)\right)\left|F\left(z_{1}^{* *}, z_{2}^{* *}, z_{3}^{*}, \ldots, z_{n}^{*}\right)\right| \leq \ldots \leq \prod_{j=1}^{n} p_{j}\left(r_{j}^{\prime}, r_{j}^{\prime \prime} \lambda_{2, j}\left(R^{\prime \prime}\right)\right) \\
\times \max \left\{\left|F\left(z_{1}, z_{2}, \ldots, z_{n}\right)\right|:\left|z_{j}-z_{j}^{0}\right|=\frac{r_{j}^{\prime}}{\lambda_{1, j}\left(R^{\prime \prime}\right) l_{j}\left(z^{0}\right)}, j \in\{1, \ldots, n\}\right\} \\
=\prod_{j=1}^{n} p_{j}\left(r_{j}^{\prime}, r_{j}^{\prime \prime} \lambda_{2, j}\left(R^{\prime \prime}\right)\right) M\left(\frac{R^{\prime}}{\Lambda_{1}\left(R^{\prime \prime}\right) \mathbf{L}\left(z^{0}\right)}, z^{0}, F\right) .
\end{gathered}
$$

Hence, by Theorem 3.1 the function $F$ is of bounded $\mathbf{L}$-index in joint variables.

\section{References}

[1] Bandura, A., Skaskiv, O., Entire functions of several variables of bounded index, Lviv, Publisher I.E. Chyzhykov, 2016, 128 p.

[2] Bandura, A.I., Skaskiv, O.B., Directional logarithmic derivative and the distribution of zeros of an entire function of bounded L-index along the direction, Ukrainian Mat. J., 69(2017), no. 3, 500-508.

[3] Bandura, A., Skaskiv, O., Analytic in the unit ball functions of bounded L-index in direciton, (submitted to Rocky Mountain Journal of Mathematics).

[4] Bandura, A.I., Bordulyak, M.T., Skaskiv, O.B., Sufficient conditions of boundedness of L-index in joint variables, Mat. Stud., 45(2016), no. 1, 12-26.

[5] Bandura, A., New criteria of boundedness of $\mathbf{L}$-index in joint variables for entire functions, (Ukrainian), Math. Bull. Shevchenko Sci. Soc., 13(2016), 58-67.

[6] Bandura, A.I., Petrechko, N.V., Skaskiv, O.B., Analytic functions in a polydisc of bounded L-index in joint variables, Mat. Stud., 46(2016), no. 1, 72-80.

[7] Bandura, A.I., Petrechko, N.V., Skaskiv, O.B., Maximum modulus in a bidisc of analytic functions of bounded L-index and an analogue of Hayman's Theorem, Mathematica Bohemica, doi: 10.21136/MB.2017.0110-16 (in print).

[8] Bandura, A., Skaskiv, O., Functions analytic in a unit ball of bounded L-index in joint variables, J. Math. Sci. (N.Y.), 227(2017), no. 1, 1-12.

[9] Bandura, A., Skaskiv, O., Filevych, P., Properties of entire solutions of some linear PDE's, J. Appl. Math. Comput. Mech., 16(2017), no. 2, 17-28.

[10] Chakraborty, B.C., Chanda, R., A class of entire functions of bounded index in several variables, J. Pure Math., 12(1995), 16-21.

[11] Fricke, G.H., Entire functions of locally slow growth, J. Anal. Math., 28(1975), no. 1, 101-122. 
[12] Hayman, W.K., Differential inequalities and local valency, Pacific J. Math., 44(1973), no. $1,117-137$.

[13] Krishna, G.J., Shah, S.M., Functions of bounded indices in one and several complex variables, In: Mathematical Essays Dedicated to A.J. Macintyre, Ohio Univ. Press, Athens, Ohio, 1970, 223-235.

[14] Kushnir, V.O., Sheremeta, M.M., Analytic functions of bounded l-index, Mat. Stud., 12(1999), no. 1, 59-66.

[15] Kushnir, V.O., Analogue of Hayman's theorem for analytic functions of bounded l-index, (Ukrainian), Visn. Lviv Un-ty, Ser. Mekh.-Math., 53(1999), 48-51.

[16] Nuray, F., Patterson, R.F., Entire bivariate functions of exponential type, Bull. Math. Sci., 5(2015), no. 2, 171-177.

[17] Nuray, F., Patterson, R.F., Multivalence of bivariate functions of bounded index, Matematiche (Catania), 70(2015), no. 2, 225-233.

[18] Patterson, R.F., Nuray, F., A characterization of holomorphic bivariate functions of bounded index, Math. Slovaca, 67(2017), no. 3, 731-736.

[19] Salmassi, M., Functions of bounded indices in several variables, Indian J. Math., 31(1989), no. 3, 249-257.

[20] Sheremeta, M.M., Entire functions and Dirichlet series of bounded l-index, Russian Math. (Iz. VUZ), 36(1992), no. 9, 76-82.

[21] Sheremeta, M.M., Analytic functions of bounded index, Lviv, VNTL Publishers, 1999.

[22] Strochyk, S.N., Sheremeta, M.M., Analytic in the unit disc functions of bounded index, (Ukrainian), Dopov. Akad. Nauk Ukr., 1(1993), 19-22.

Andriy Bandura

Ivano-Frankivsk National Technical University of Oil and Gas

Department of Advanced Mathematics

15, Karpatska Street, 76008 Ivano-Frankivsk, Ukraine

e-mail: andriykopanytsia@gmail.com

Oleh Skaskiv

Ivan Franko National University of Lviv

Department of Function Theory and Theory of Probability

1, Universytetska Street, 79000 Lviv, Ukraine

e-mail: olskask@gmail.com 